$$
\text { CONF-980607--1 }
$$

\title{
Multivariate Prediction: Selection of the Most Informative Components to Measure
}

\author{
S. Batsell * \\ V. Fedorov ** \\ D. Flanagan $* *$
}

* Network Research Group Mathematical Sciences Section

** Statistics Group

Mathematical Sciences Section

Oak Ridge National Laboratory

Computer Science and Mathematics Division

P. O. Box 2008, Bldg. 6012

Oak Ridge, TN 37831-6367

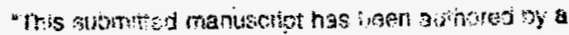
contractor of the U.S. Government under Contract No. DE-AC05-96OR22464. Accordingly, the U.S. Govemment retains a nonexclusive, royalty-frea license to publish or reproduce the published form of this contribution, or allow others to do so, for U.S. Government purposes."
}
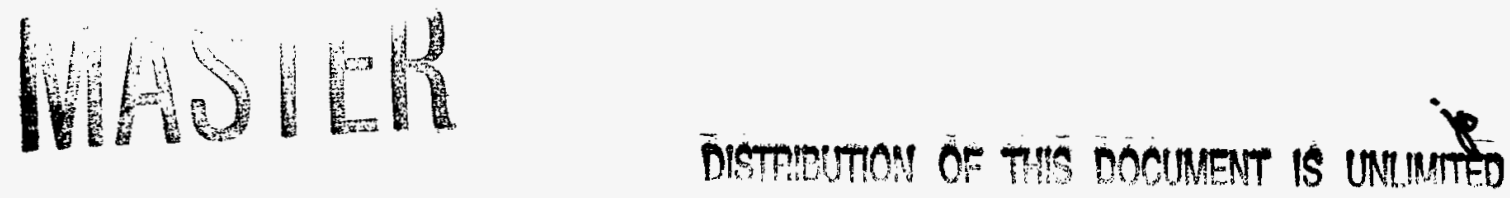

Research sponsored by the Office of Energy Research, U.S. Department of Energy under contract number DE-AC05-96OR22464 with Lockheed Martin Energy Research Corporation.

\section{3}




\title{
Multivariate Prediction: Selection of the Most Informative Components to Measure.
}

\author{
Batsell S., Fedorov V., Flanagan D. \\ Oak Ridge National Laboratory \\ Oak Ridge, Tennessee 37831, U.S.A.
}

\begin{abstract}
A number of interesting problems in the design of experiments such as sensor allocation, selection of sites for the observing stations, determining sampler positions in traffic monitoring, and which variables to survey/measure in sampling studies may be considered in the following setting: Given a covariance matrix of multi-dimension random vector and given a ratio of the number of possible observations to the observational error select those components which must be observed to guarantee minimization of an objective function describing the quality of prediction of all or prescribed components. We show that the problem can be considered in the framework of convex design theory and derive some simple but effective algorithm for selection of an optimal subset of components to be observed.
\end{abstract}

Key words: Design of experiments; Prediction; Correlated observations.

\section{Optimality criteria.}

Model and main assumptions. Let $U$ be a random vector which we are interested in and let its components be measured with some random error:

$$
Y=U+\mathcal{E},
$$

where $Y, U, \mathcal{E} \in R^{s}, E(U)=U_{0}, \operatorname{Var}(U)=K, E(\mathcal{E})=0, \operatorname{Cov}(\mathcal{E})=\Sigma$, $E\left(\mathcal{E} U^{T}\right)=0$.

In what follows the same characters are used for random variables and their realizations. The latter ones are usually marked with some subscripts. The low case characters are reserved for the vector components. For instance, $U=\left(u_{1}, \ldots, u_{s}\right)^{T}$. The matrix $\Sigma$ is diagonal and

$$
\xi=\left\{p_{i}\right\}_{1}^{s}, \quad p_{i}=r_{i} / N
$$


is called a design (of an experiment). Some of its components can be equal zero.

Estimation and criteria of optimality. Let $\bar{Y}_{j}(\xi)=\left(\bar{y}_{i j}(\xi), \ldots, \bar{y}_{s j}(\xi)\right)$ be the vector of averaged observations given the $j$-th realization of $U$, i.e.

$$
\bar{y}_{i j}(\xi)=\frac{1}{r_{i}} \sum_{k=1}^{r_{i}} y_{i j k},
$$

and let (compare with (2))

$$
\Sigma(\xi)=W^{-1}(\xi)=\sigma^{2} / N p_{i}
$$

It can be verified that the estimator (the approach can be similar to Rao (1973), Chpt.4i):

$$
\hat{U}(\xi)=K^{T}(K+\Sigma(\xi))^{-1} \bar{Y}(\xi)
$$

minimizes the variance of prediction

$$
E_{u, \varepsilon}\left[\left(L^{T} \tilde{U}(\xi)-L^{T} U\right)^{T}\right]=\operatorname{Var}\left(L^{T} \tilde{U}(\xi)\right)
$$

for any linear function $L^{T} U$ among all linear estimators $\tilde{U}(\xi)$ such that

$$
E_{u, \varepsilon}\left(L^{T} \tilde{U}(\xi)-L^{T} U\right)=0 .
$$

The indices $u$ and $\varepsilon$ indicate variables with respect to which the expectation must be taken.

From (1) and (3) it follows that

$$
\operatorname{Var}(\hat{U}(\xi))=D(\xi)=K-K(K+\Sigma(\xi))^{-1} K
$$

In (3) and (6) it was assumed that all $p_{i}>0$. If some of weights $p_{i}$ are zeros, i.e., some of components of $U$ are not measured, then (3) and (6) are to be replaced by

$$
\hat{U}\left(\xi_{n}\right)=\underline{K}^{T}\left(\xi_{n}\right)\left(K\left(\xi_{n}\right)+\Sigma\left(\xi_{n}\right)\right)^{-1} \bar{Y}\left(\xi_{n}\right)
$$

and

$$
D\left(\xi_{n}\right)=K-\underline{K}^{T}\left(\xi_{n}\right)\left(K\left(\xi_{n}\right)+\Sigma\left(\xi_{n}\right)\right)^{-1} \underline{K}\left(\xi_{n}\right) .
$$

Correspondingly now $\xi_{n}$ is a collection of $n$ nonzero weights; $\Sigma\left(\xi_{n}\right)$ is an $n \times n$ diagonal matrix and $\Sigma_{i i}\left(\xi_{n}\right)=\sigma^{2} / N p_{i}, p_{i}>0$; the $n \times n$ matrix $K\left(\xi_{n}\right)$ is obviously defined a submatrix of $K$; the $n \times s$ matrix $\underline{K}\left(\xi_{n}\right)$ is a submatrix of $K$ which describes covariances between the measured components and all 
others (including the measured ones). Note that, as before, $\hat{U}\left(\xi_{n}\right)$ has $s$ components and $D\left(\xi_{n}\right)$ is an $n \times n$ matrix. Formulae (7) and (8) simplify computations and help to avoid any limit transitions when some of weights in $\xi$ vanish.

For the theoretical exercises it is more convenient to use the following presentation of the covariance matrix of prediction $\hat{U}(\xi)$ :

$$
D(\xi)=\left(K^{-1}+W(\xi)\right)^{-1}, W^{-1}(\xi)=\Sigma(\xi) .
$$

To derive (9) from (6) one may use the identity $(A+B)^{-1}=A^{-1}-A^{-1}\left(A^{-1}+\right.$ $\left.B^{-1}\right)^{-1} A^{-1}$. From the fact that the estimator $\hat{U}(\xi)$ minimizes the variance of prediction of any linear function $L^{T} U$ it follows that

$$
D(\xi)=\operatorname{Var}(\hat{U}(\xi)) \leq \operatorname{Var}(\tilde{U}(\xi)),
$$

where $\tilde{U}(\xi)$ is defined as in (3) and (4). Hence, the same estimator minimizes any function $\Psi(D(\xi))$ which is monotonic, i.e., $\Psi(A) \leq \Psi(B)$, where $A$ and $B$ are nonnegative definite matrices and $A \leq B$.

The next step, which is expedient to consider, is to find

$$
\xi^{*}=\arg \min _{\xi} \Psi(D(\xi)) .
$$

The choice of the function $\Psi$ is discussed in any book on experimental design (c.f. Fedorov (1972), Pazman (1986), Pukelsheim (1993)). In this paper we confine ourselves to D-optimality and linear optimality:

$$
\Psi(D)=\ln |D| \text { and } \Psi(D)=\operatorname{tr} A D,
$$

where $A$ is a given nonnegative definite matrix.

\section{Continuous optimal weights.}

Optimization problem (10) can be considered as a particular case of convex design theory. Two specific features of (10) deserve to be mentioned. First, the matrix $D(\xi)$ in many applications can be relatively large. Frequently $s$ can equal a few hundreds. Second, the dependence of $D(\xi)$ upon $\xi$ or $p_{i}, i=$ $1, \ldots, s)$ is very simple compared to what is usually seen in optimal design of experiments.

The following results are direct corollaries of the generalized version of the equivalence theorem and the convexity of the matrix $D(\xi)$ :

$$
D\left[(1-\alpha) \xi_{1}+\alpha \xi_{2}\right] \leq(1-\alpha) D\left(\xi_{1}\right)+\alpha D\left(\xi_{2}\right) .
$$


The inequality $A \leq B$ means that $C^{T} A C \leq C^{T} B C$ for any vector $C$. For our current objectives the most convenient for our current objectives form of this theorem can be found in Fedorov (1996).

Theorem 1 A necessary and sufficient condition for $\xi^{*}$ to be D-optimal is that

$$
D_{i i}\left(\xi^{*}\right) \leq \frac{\sigma^{2}}{N} \operatorname{tr} W\left(\xi^{*}\right) D\left(\xi^{*}\right), i=1, \ldots, s,
$$

and the equality holds at all points where $p_{i}^{*}>0$.

$A D$-optimal design minimizes the maximal variance of prediction, i.e.

$$
\xi^{*}=\arg \min _{\xi} \max _{i} D_{i i}\left(\xi^{*}\right) .
$$

Theorem $2 A$ necessary and sufficient condition for $\xi^{*}$ to be linear optimal is that

$$
\sum_{j=1}^{s}\left\{D\left(\xi^{*}\right) A D\left(\xi^{*}\right)\right\}_{i j} \leq \frac{\sigma^{2}}{N} \operatorname{tr} W\left(\xi^{*}\right) D\left(\xi^{*}\right) A D\left(\xi^{*}\right), i=1, \ldots, s
$$

and the equality holds at all points where $p_{i}^{*}>0$.

Unlike most cases in the traditional experimental design, the value of the optimal weights $p_{i}^{*}$ depends upon the total number of available observations. If $A=I$, then the average variance of the prediction is minimized. Selecting

$$
A=\left(\begin{array}{cc}
I_{q} & 0 \\
0 & 0
\end{array}\right)
$$

we minimize the average variance of prediction of $q$ out of $s$ components.

\section{Numerical construction of $\xi^{*}$.}

As it has been already mentioned the dimension $s$ of the vector $U$ can be very significant, and, therefore, any numerical procedure for construction of $\xi^{*}$. must process (and, in particular, to invert) matrices of large sizes. Therefore, it is important to develop algorithms which are based on simple recursions. The following algorithm closely resemble the exchange type algorithms developed in the traditional design theory (c.f. Fedorov (1996), Mitchell (1974)). We describe here only the simplest version of the algorithm for D-criteria.

Let $\xi_{0}$ have weights $p_{0 i}=b_{i} \alpha_{0}$, where $\dot{b}_{i}$ are integer and $\sum_{i=1}^{s} p_{0 i}=1$. 
Step (a). Given $\xi_{t}$ find

$$
a=\arg \min _{i} D_{i i}\left(\xi_{t}\right)
$$

Increase the weight $p_{a}$ by $\alpha_{t}$ to construct $\xi_{t}^{+}$.

Step (b). Find

$$
d=\arg \min _{i \in J_{t}} D_{i i}\left(\xi_{t}^{+}\right),
$$

where $J_{t}$ is the current set of component with nonzero weights. Subtract $\alpha_{t}$ from $p_{d}$. Call the modified design $\xi_{t+1}$.

Step (c). If

$$
\ln \left|D\left(\xi_{t}\right)\right|-\ln \left|D\left(\xi_{t+1}\right)\right| \leq \gamma,
$$

where $\gamma$ is a small positive constant, then let $\alpha_{t+1}=\alpha_{t} / 2$ and process steps (a) and (b) with $\xi_{t+1}$. Otherwise, use $\alpha_{t+1}=\alpha_{t}$ in processing steps (a) and (b) with $\xi_{t+1}$.

Theorem 3 The sequence $\left\{\left|D\left(\xi_{t}\right)\right|\right\}$ converges and

$$
\lim _{t \rightarrow \infty} \ln \left|D\left(\xi_{t}\right)\right|-\min _{\xi} \ln |D(\xi)| \leq \gamma .
$$

The above result may be proved using the technique developed in convex design theory (c.f. Cook and Fedorov (1995)). The partitioning rule at step (c) may be replaced by another one:

$$
D_{a a}\left(\xi_{t}\right)-D_{a a}\left(\xi_{t+1}\right) \leq \delta
$$

where $\delta$ is a small positive constant.

To avoid the inversion of large size matrices at every step of the above iterative procedure the following recursions may be used for the forward steps:

$$
\left|D\left(\xi_{t}^{+}\right)\right|=\frac{\left|D\left(\xi_{t}\right)\right|}{1+\zeta_{t} D_{a a}\left(\xi_{t}\right)}, D\left(\xi_{t}^{+}\right)=D\left(\xi_{t}\right)-\frac{\zeta_{t} C^{+}\left(\xi_{t}\right)}{1+\zeta_{t} D_{a a}\left(\xi_{t}\right)},
$$

where $\zeta_{t}=N \alpha_{t} / \sigma^{2}$ and $C_{i j}^{+}=D_{a i} D_{a j}$. For the backward steps the sign of $\zeta_{t}$ must be changed.

Example. Let

$$
K_{i j}=0.02 \min (i, j)(50-\max (i, j)), i, j=1, \ldots, 49 .
$$

We computed D-optimal designs for $\sigma^{2} N^{-1}=0.01,0.1$ and 1. The results (after 100 iterations and $\delta=0.001$ ), are exhibited exhibited at Fig. 1 and 2. 


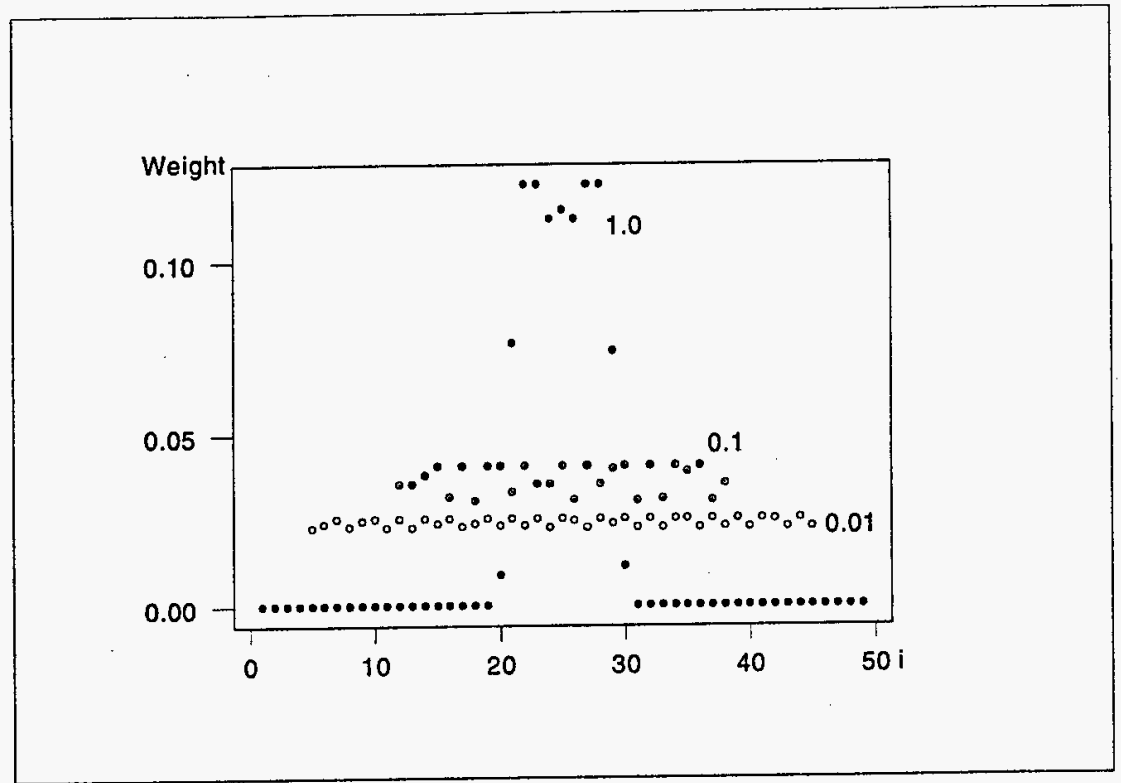

Figure 1: Computed weights for $\sigma^{2} N^{-1}=0.01,0.1$ and 1

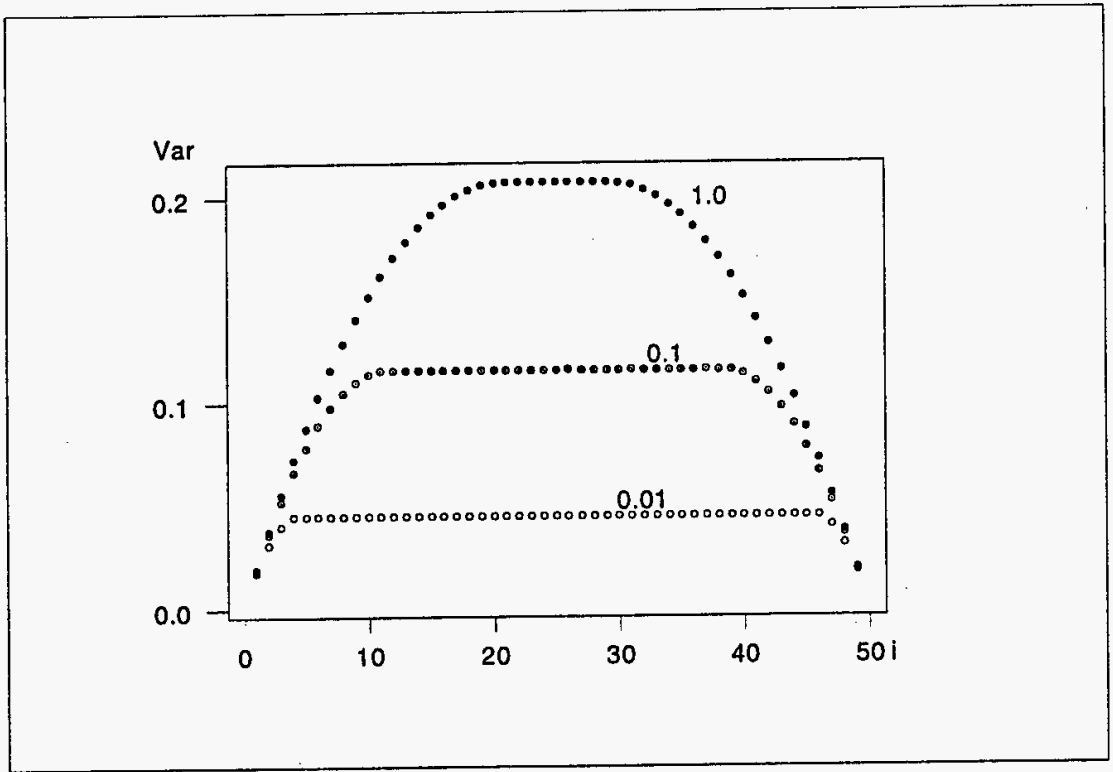

Figure 2: Variance of prediction for $\sigma^{2} N^{-1}=0.01,0.1$ and 1 
The weights were not rounded up or symmetrized with respect to $i=25$, and that is an obvious step in more practical situations.

In this example we used the iterative procedure without any modification. Actually we found that some minor amendments improve the rate of convergence significantly. For instance, instead of proceeding with one step forward - one step backward, one can repeat a few steps forward and then exactly the same number of steps backward (to keep $\sum_{i=1}^{s} p_{i}=1$ ). Instead of using the constant $\gamma$ or $\delta$, some slowly diminishing sequences $\left\{\gamma_{t}\right\}$ or $\left\{\delta_{t}\right\}$ do a better job, etc.

\section{Areas of application.}

Population sampling. Let components of the vector $U_{j}$ correspond to some economic or social quantitative characteristic for $s$ different population groups. The subscript $j$ can indicate, for instance, that $U_{j}$ is a realization of the random vector $U$ corresponding either to the $j$-th moment of time or to the $j$-th region. From historical data we can evaluate $C o v U$. Given a total number $N$ of observations we have to define $r_{i}^{*}=p_{i}^{*} N$ to make the results of survey study most accurate (c.f. Wynn (1982)).

Measurement of traffic intensities. Let the traffic system be described by a graph with $s$ edges. These edges are roads in the conventional traffic problem or links in the communication network problem. What are the optimal fractions $r_{i}^{*}=p_{i}^{*} N$ of the total number of available observation which must be done (if any) at each $i$-th edge for the better prediction of traffic intensities $U_{i j}$ on a particular $j$-th day (hour, minute, ...)? One can measure traffic intensities, delay times, lengths of queues, etc. (c.f. Frost and Melamed (1994)).

Sensors allocation. Assume that some pollution or meteorological parameters must be predicted at $s$ various parts of some region. How many sensors out of a total $N$ must be allocated at each subregion or how many repeated observations with one sensor must be done (c.f. Cressie (1991))? If the covariance structure of $U$ is known then we can apply the proposed approach.

\section{Acknowledgment.}

This study was supported by the Oak Ridge National Laboratory Directed Research and Development Fund. 


\section{References}

[1] Cook R.D. and Fedorov V. V. (1995). "Constrained Optimization of Experimental Design". Statistics, 26, 129-178.

[2] Cressie N.A. (1991). Statistics for Spatial Data, Wiley, New York.

[3] Fedorov V.V. (1971). Theory of Optimal Experiments, Academic Press, New York.

[4] Fedorov V.V. (1996). "Design of Spatial Experiments: Model Fitting and Prediction". In Ghosh S. and Rao C.R. (Eds.), Design and Analysis of Experiments, Handbook of Statistics, Vol. 13, pp. 515-553.

[5] Frost, V.S. and Melamed B. (1994). "Traffic Modeling for Telecommunications Networks", IEEE Communications Magazine, March (1994).

[6] Pazman A. (1986). Foundation of Optimum Experimental Design. Reidel, Dordrecht.

[7] Puckelsheim F. (1993). Optimal Design of Experiments. Wiley, New York.

[8] Rao C. R. (1973). Linear Statistical Inference and its Applications, 2nd Edition, Wiley, New York.

[9] Wynn H.P. (1982). Optimum Submeasures with Applications to Finite Population Sampling. In Statistical Decision Theory and Related Topics III, Vol. 2., Academic Press, New York, pp. 485-495.

\section{DISCLAIMER}

This report was prepared as an account of work sponsored by an agency of the United States Government. Neither the United States Government nor any agency thereof, nor any of their employees, makes any warranty, express or implied, or assumes any legal liability or responsibility for the accuracy, completeness, or usefulness of any information, apparatus, product, or process disclosed, or represents that its use would not infringe privately owned rights. Reference herein to any specific commercial product, process, or service by trade name, trademark, manufacturer, or otherwise does not necessarily constitute or imply its endorsement, recommendation, or favoring by the United States Government or any agency thereof. The views and opinions of authors expressed herein do not necessarily state or reflect those of the United States Government or any agency thereof. 


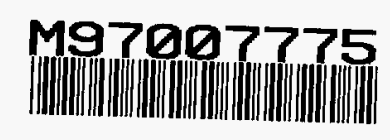

Report Number (14) CONF-980607 --1

$\begin{array}{ll}\text { Publ. Date (11) } & 199806 \\ \text { Sponsor Code (18) } & \text { DOE/ER, XF } \\ \text { UC Category (19) } & \text { UC-400,DOE/ER }\end{array}$ 